\title{
Transformación digital. El caso de la creación de una oficina virtual en una Institución de Educación Superior
}

\section{Digital transformation. The case of creating a virtual office in a Higher Education Institution}

DOI: $10.46814 /$ lajdv3n5-026

Recebimento dos originais: 01/05/2021

Aceitação para publicação: 31/06/2021

\section{Dr. Edgar René Vázquez González}

Doctor en Administración y Estudios Organizacionales, Profesor de Tiempo Completo en el Departamento de Estudios Organizacionales, de la División de Ciencias Económico Administrativas de la Universidad de Guanajuato.

Dirección postal: Sede Marfil campus Guanajuato, Fracc. El Establo, Guanajuato, Gto. C.P.:36250 Correo electrónico: edrvazquez10@gmail.com

\section{Dra. Cecilia Ramos Estrada}

Doctora en Administración y Estudios Organizacionales, Profesora de Tiempo Completo en el Departamento de Estudios Organizacionales, de la División de Ciencias Económico Administrativas de la Universidad de Guanajuato.

Dirección postal: Sede Marfil campus Guanajuato, Fracc. El Establo, Guanajuato, Gto. C.P.:36250 Correo electrónico: ceciliagto@gmail.com

\section{RESUMEN}

El presente trabajo tiene como objetivo describir el proceso de transformación digital en una oficina de servicios en una Institución de Educación Superior Pública en México, para ello se realizó un análisis de la revisión de la literatura relacionada con los conceptos: transformación digital y servicios públicos. A partir de los resultados del análisis de la literatura se identificaron las dimensiones que posibilitan la transformación digital en organizaciones del sector público. Posteriormente, en el caso de estudio se describen los principales servicios que migraron a formatos digitales y que dio origen a una ventanilla virtual de servicios de la institución, el modelo bajo el cual se desarrolló, el impacto generado en los usuarios. El trabajo utilizó una metodología cualitativa que permitió identificar las dimensiones que componen un modelo de transformación digital en instituciones del sector público y cuya viabilidad deberá ser corroborada a través de estudios con mayor profundidad y rigor metodológico.

Palabras clave: transformación digital, innovación, instituciones de educación superior pública.

\begin{abstract}
The present work aims to describe the digital transformation process in a service office in a Public Higher Education Institution in Mexico, for which an analysis of the literature review related to the topics of the fourth industrial revolution, digital transformation and public services. From the result of the analysis of the literature, the dimensions that enable digital transformation in public service organizations were identified. Later, in the case study, the main services that migrated to digital formats and that gave rise to the institution's virtual service window, the model under which it was developed, the impact generated on users are reported. The work was developed under a qualitative methodology that allowed identifying the dimensions that make up a digital transformation model in public sector
\end{abstract}


institutions and whose viability should be corroborated through studies with greater depth and methodological rigor.

Key words: Digital transformation, innovation, higher education institution.

\section{INTRODUCCIÓN}

Es indudable que la cuarta revolución industrial ha influido en la producción de bienes y servicios a nivel global. Dicha revolución se ha caracterizado por un creciente cambio tecnológico y un acelerado desarrollo de innovación Ghobakhloo (2020); Schwab (2016); Christensen; Raynor y McDonald (2015).

En esta revolución, las tecnologías emergentes y la innovación de base extendida se están difundiendo mucho más rápido y ampliamente que en las anteriores revoluciones (Ye y Kankanhalli, 2020). La escala y el alcance del cambio explican las razones de que la disrupción e innovación están presentes de manera acelerada hoy en día (Karlsson, Larsson, Ronnback, 2018). Algunas de las aplicaciones derivadas de este cambio tecnológico en las empresas e instituciones son, el internet de las cosas (IoT), la inteligencia artificial, robótica, el Big Data, el cloud, la TV4K, los teléfonos móviles 5G, el Blockchain, la realidad virtual y aumentada, los drones, bitcoins, geolocalización, la ciberseguridad, las impresoras 3D, la nanotecnología, entre otros (Schwab, 2016). Lo que es cierto es que dichas aplicaciones han propiciado el desarrollo de nuevas formas de trabajo, nuevos modelos de negocio, y nuevas formas de convivencia; este avance tecnológico ha permeado a las Instituciones de Educación Superior, lo que ha implicado generar aplicaciones para el desarrollo de sus actividades docentes, de investigación, de extensión y de gestión (Castro, L, J. Tamayo, et. al. 2020)

La influencia, magnitud y la rapidez de la implementación de estas aplicaciones han transformado también a las Tecnologías de la Información y la Comunicación (TIC’S), ello ha configurado el concepto Revolución Digital (Cuenca-Fontbona, et al., 2020). De acuerdo con la (OCDE, 2019) "la digitalización es una tecnología de utilidad general que da soporte a todos los sectores productivos". En el escenario global se caracteriza por el intercambio de información (programas, servicios, aplicaciones), de ideas que han evolucionado al mercado y a los negocios, así como al trabajo y las relaciones sociales.

El uso del internet en las organizaciones ha reformado los procesos y los modelos de organización, que permiten una mejor comunicación, generando información en tiempo real, ello facilita la toma de las decisiones. La transformación digital que hoy impera trasciende y modifica los hábitos de las personas en todos los aspectos de la vida, y de igual forma en las empresas e instituciones (Weill y Woerner, 2018). 
La finalidad del trabajo es describir el proceso de transformación digital que dio origen a una ventanilla virtual de servicios en Recursos Humanos en una Institución de Educación Superior Pública en México; se hizo una revisión sistemática de la literatura del tema, producto de este análisis se identificaron los modelos que coadyuvan a las organizaciones del sector público a realizar de manera más efectiva este tipo de transformaciones. Se identificaron tres dimensiones: la primera es estrategia y liderazgo; la segunda las capacidades digitales que debe tener una institución; y la tercera es la gestión del cambio (Mergel, 2018).

El trabajo tiene un enfoque de tipo cualitativo utilizando el caso de estudio que relata la manera en que se realizó el proceso de transformación digital en la institución educativa estudiada. El presente estudio pretende dejar líneas de reflexión para el desarrollo de estudios que permitan explicar el espectro de fenómeno desde una perspectiva de la gestión y la innovación.

\section{REVISIÓN DE LA LITERATURA}

La Transformación Digital se define como "el proceso de gestión que orienta la cultura, la estrategia, las metodologías y las capacidades de una organización a partir de las tecnologías digitales" (Cuenca-Fontbona, et al., 2020, p.78). Por su parte (Gooble, 2018, p.66) define:

\footnotetext{
"Transformación digital como la profunda transformación de las actividades y organizaciones empresariales, procesos, competencias y modelos, para la máxima transformación de los cambios y oportunidades con una combinación tecnológica y su impacto acelerado en la sociedad, de una manera estratégica y prioritaria".
}

En estos conceptos se destaca que las organizaciones deben contemplar a la transformación desde la estrategia y como práctica prioritaria.

La transformación digital sucede cuando una organización reemplaza sus productos o servicios, a través de la conexión inteligente entre ellos y las personas, o de la mezcla de productos físicos y servicios virtuales (Lombardero, 2015). La extensión de la tecnología ha propiciado escenarios en el que la conectividad y los dispositivos mediante los cuales se desarrolla la digitalización permean a todos los aspectos de la vida de las personas. Incluso en el servicio público cada vez las instituciones de gobierno incorporan la tecnología para brindar servicios digitales a sus ciudadanos. (Jannsen y Estévez, 2013)

Así, la multiplicación de las demandas de los servicios, la distancia, la regionalización, la velocidad de la transmisión, la convergencia de diferentes tecnologías, así como la capacidad de reaccionar rápidamente han llevado a los dirigentes de las organizaciones a perfeccionar la atención al cliente a través de sistemas interactivos multimedia (Mergel, I. 2018; Hofmann, et.al. 2018). 


\section{CONTEXTO NACIONAL EN TRANSFORMACIÓN DIGITAL EN SERVICIOS} PÚBLICOS.

Para el caso de México en el año 2019 se ubicó en la posición 49 del Índice Mundial de Competitividad Digital de la IMD Business School, el cual evalúa a 63 países sobre cómo adoptan y exploran las tecnologías digitales para transformar prácticas de Gobierno, modelos de negocio y a la sociedad en general. En este mismo reporte se destaca que: “a esta nación lo ubican en el número 50 en el aspecto del uso de las tecnologías digitales disponibles sean absorbidas por la sociedad" (Hernández, 2019). Sin embargo, es importante reconocer que en los últimos diez años tuvo un dinamismo importante en la adopción y uso de Tecnología, pero los retos aún son grandes para el país.

Este tipo de índices toman en consideración las siguientes variables: el costo por el uso de conexión a internet, confiabilidad de la infraestructura; acceso a redes; capacidad, que se refiere al ancho de banda para cada usuario y velocidad de conexión; el uso que es la actividad de internet minorista, gobierno electrónico, penetración de internet, usuarios de redes sociales; y capital humano (Cave, Flores-Rouux, 2017).

La digitalización para el gobierno y los servicios públicos, de acuerdo con (WBR, 2016) "las tecnologías digitales han ayudado a los gobiernos a la entrega eficiente del servicio que requiere un gobierno capaz, que pueda implementar políticas y derogar los recursos públicos de manera efectiva”. La transformación digital en el sector público significa nuevas formas de trabajar con las partes interesadas, construir nuevos marcos de servicios y crear nuevas formas de relación entre sociedad y gobierno. (Mergel, Edelman, Haug, 2019). Lo anterior permite brindar mejores servicios a los ciudadanos, y desde luego incidir de manera favorable en materia de transparencia y rendición de cuentas, porque disminuye la intervención de las personas en los procesos del gobierno (Skalen, Karlsson, et.al., 2017).

Adicionalmente la generación de datos que se obtiene producto de transformar a modo digital los servicios, que a través de los repositorios donde se almacena la información, les permite a las instituciones contar con volúmenes de datos para análisis, tomas de decisiones y generación de políticas públicas en beneficio de los ciudadanos (Dalkir, 2017).

Ciertamente, se requiere definir una estrategia digital del gobierno y un plan para su implementación. Para el caso de México en el año 2013 la reforma a la Ley Federal de Telecomunicaciones y Radiodifusión consideró que el estado tiene la obligación constitucional de garantizar el acceso a las TIC para todos los mexicanos. Desde ese año, el gobierno en turno lanzó la Estrategia Digital Nacional, y en el gobierno actual se continúa con dicha estrategia denominada México Digital que establece "un plan de acción en el que la tecnología y la innovación contribuyan a alcanzar las grandes metas de desarrollo del país". Dicha estrategia está compuesta por 5 objetivos 
(Gobierno de México, 2020). Cada uno de los objetivos cuentan con sus acciones y metas, sin embargo, existen críticas por parte de los ciudadanos en el sentido de que dichas estrategias y acciones se encuentran desarticuladas, poco coordinadas y los planes suelen perderse.

A efecto de contar con una adecuada transformación digital en las organizaciones, se requieren de dos condiciones: a) el liderazgo (con visión digital) es decir, la intensidad de la gestión transformacional o el empuje, la convicción y la creencia por parte del gobierno o de la organización en una firme apuesta de valor diferencial y beneficiosa para el consumidor; y b) las capacidades digitales, que son los recursos tecnológicos que las organizaciones invierten para cambiar su gestión operativa y aumentar su eficiencia, así como, la competitividad (Cuenca-Fontbona, Matilla y ComptePujol, 2020).

Las condiciones señaladas en el párrafo anterior son fundamentales para que la estrategia digital se impregne en la organización y en sus procesos, ambas se requieren en la misma intensidad, la ausencia o disminución de alguna obstaculizará el proceso de transformación digital. Considerando lo anterior, con la revisión de la literatura, se identificó que se han desarrollado modelos de transformación, los propios autores Westerman, McAfee y Bonnet (2014) propusieron un modelo que se ha aplicado en muchas empresas con resultados favorables, sin embargo, y dado que el enfoque que se presenta en este trabajo está orientado al servicio en instituciones públicas, se revisaron varios modelos más, que se adapten mejor a la organización estudiada.

\section{MODELO DE TRANSFORMACIÓN DIGITAL.}

La transformación digital es un proceso que reduce actividades manuales y de bajo valor, genera innovadores servicios digitales y una satisfacción en los clientes (Wang y Li, 2019). Además, habilita nuevas formas de trabajar y permite a las empresas ser más ágiles, rápidas y eficientes. La propuesta de modelo de transformación digital de la organización española nae (2018) contempla los siguientes enfoques: liderazgo y capacidad digital, y agrega el cambio operativo, basado en la estrategia de la organización orientada a los procesos.

El modelo que plantea nae se compone de un punto central (BASICS) compuesto de ocho variables como se muestra en 1 siguiente figura: 
Figura 1. Modelo de transformación digital.

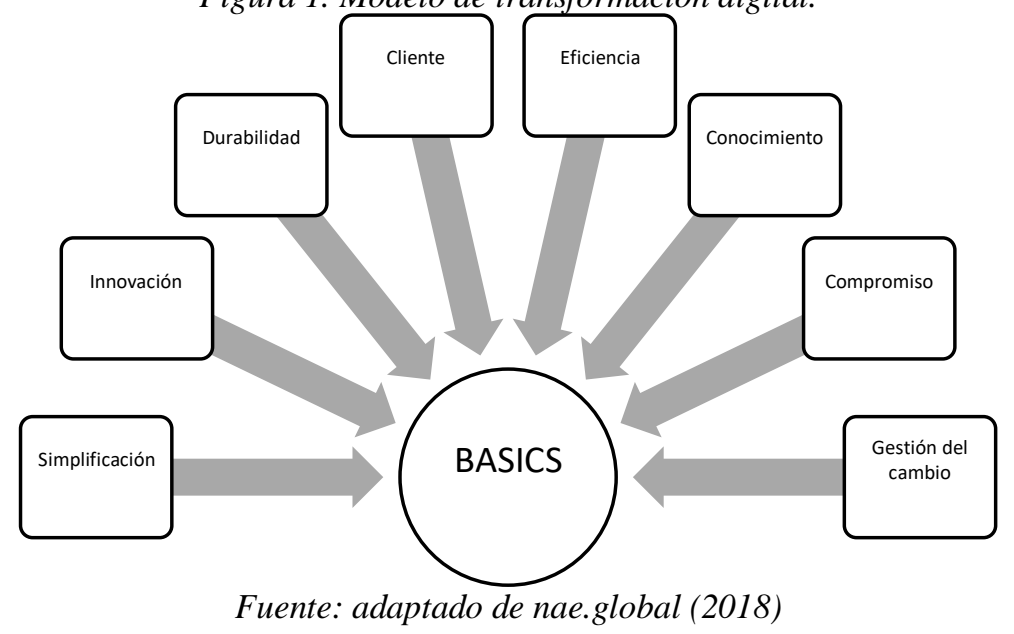

Este modelo plantea como punto de arranque la estrategia digital y las capacidades tecnológicas, que se relacionan con las siguientes variables:

- Cliente: es lo primero, y no es un slogan, es atender las necesidades y brindar el servicio con calidad.

- Durabilidad: los nuevos modelos deben ser válidos a largo plazo.

- Innovación: no vale repetir lo que ya existe, pensar en agregar valor.

- Simplificación: hacer las cosas de la forma más sencilla.

- Eficiencia: el camino más corto y optimizando los recursos.

- Conocimiento: el bien más preciado que debe quedarse dentro de la organización

- Compromiso: la alta dirección debe liderar, motivar y participar

- Gestión del Cambio: la gente debe creer.

En este modelo, la transformación digital es una gran oportunidad que se articula y vertebra en tres piezas: i) Rediseño de procesos de extremo a extremo con foco en su automatización; ii) Explotar los datos para automatizar operaciones y procesos del negocio, y iii) Self-everything: autodiagnóstico, autogestión, autorecovery (Nae, 2018).

Dicho modelo tiene el potencial de incidir para la transformación digital de gobierno y servicios públicos; las variables que se señalan configuran el modelo que permite lograr el objetivo de utilizar la tecnología para mejorar la relación con su gente.

\section{MÉTODO}

Para el desarrollo de este trabajo se utilizó una investigación tipo cualitativa, utilizando la revisión sistemática de la literatura a partir de la propuesta Ding et. al. (2014) que ayudó a identificar los trabajos realizados sobre la cuarta revolución industrial y transformación digital en el ámbito 
público. A efecto de realizar el análisis se utilizaron plataformas de búsqueda de documentos indizados y de factor de impacto, tal es el caso de Elsevier Scopus, y se utilizó el software Harzing, AW (2007) Publish or Perish, dicho programa permite recuperar y analizar documentos, así como, citas académicas, además de que facilita realizar análisis y obtener métricas. De esta manera en las plataformas de búsqueda se plasmaron los conceptos "cuarta revolución industrial” y "transformación digital" y "servicios públicos". El rango de datos fue de "todos los años" a la "fecha", así como por todas las áreas del conocimiento.

Del total de los datos arrojados, se aplicó un filtro a efecto de seleccionar los recursos más relevantes al tema central de este trabajo. Los criterios utilizados para la selección fueron: a) artículos, conferencias de congresos o capítulos de libro; b) número de citas; y c) que los artículos contengan las dimensiones estrategia, capacidades digitales, gestión y cambio. El resultado del análisis se presenta más adelante.

Una vez realizado lo anterior, se utilizó la metodología caso de estudio de tipo explicativo (Yin,1984), para estudiar el proceso de transformación digital a través de la creación de una ventanilla virtual de servicios en una oficina de recursos humanos en una Institución de Educación Superior en México. Para realizar el caso, se solicitó la autorización de la alta dirección, teniendo la anuencia para realizar el estudio. Posteriormente se solicitaron documentos (Programas y planes de trabajo institucionales, proyecto de desarrollo tecnológico, entre otros) y se aplicaron entrevistas, principalmente a los actores que participaron en el proyecto. Los criterios seleccionados para desarrollar el caso, se obtuvieron a partir de la revisión de la literatura, siendo estos: estrategia, capacidades digitales, gestión y cambio. En el apartado caso de estudio se describen los resultados identificados.

\section{ANÁLISIS DE LOS DATOS A PARTIR DE LA REVISIÓN DE LA LITERATURA.}

A continuación, se presenta el análisis utilizando los conceptos "cuarta revolución industrial" y “transformación digital”. El resultado arrojó 115 documentos, publicados a partir del año 2016 al año 2020. El mayor número de artículos publicados se dio a partir del año 2018 a la fecha, en este periodo de tiempo acumulan 108 documentos. Los países que más publicaron fueron: Rusia, Alemania, Sudáfrica, Italia, España y Estados Unidos. Para el caso de México solo se ubicaron dos artículos.

En los siguientes gráficos se muestran los resultados de tipo de documento y el área de conocimiento donde se publicaron. 
Figura 2. Documentos relacionados con los conceptos “Cuarta Revolución Industrial” y “Transformación Digital”.

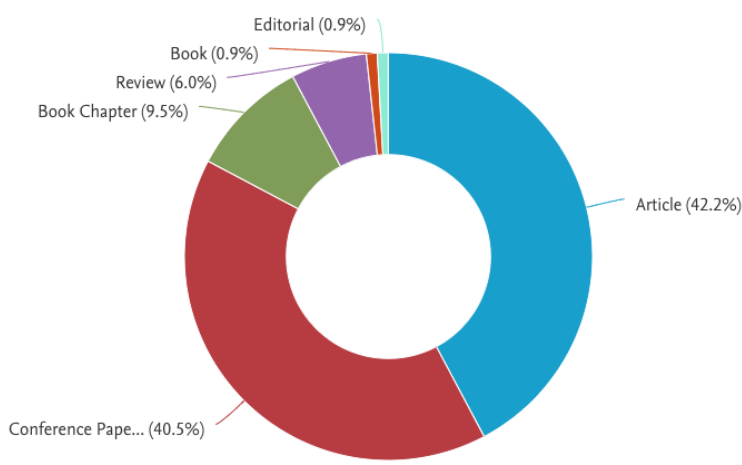

Fuente: Scopus (2020)

Se puede apreciar en la figura 2 , que el $42.2 \%$ de los documentos relacionados con los conceptos del objeto de estudio son artículos, mientras que el $40.5 \%$ son ponencias en conferencias y el $9.5 \%$ capítulo de libro; de estos resultados se tomaron como fuente para este estudio los artículos y capítulos de libro.

Figura 3. Documentos relacionados con los conceptos "Cuarta Revolución Industrial” y "Transformación Digital" por áreas de conocimiento.

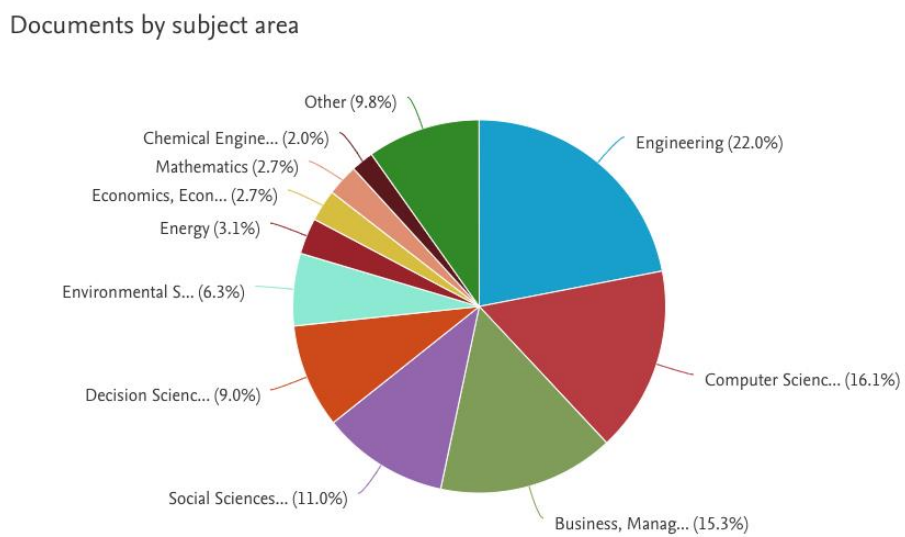

Fuente: Scopus (2020)

De la anterior figura se destacan los resultados arrojados de la búsqueda por áreas de conocimiento, el $38.1 \%$ de los documentos están asociados a las áreas de ingenierías y ciencias de la computación, mientras que $26.3 \%$ corresponden a las áreas de negocios, administración y las ciencias sociales que abordan estos conceptos; lo anterior coadyuva para seleccionar los documentos que abordan las dimensiones de análisis: estrategia, capacidades digitales, gestión y cambio. 
En lo que respecta a la búsqueda de documentos con los conceptos “Transformación Digital” y “Servicios Públicos" se encontraron 100 documentos publicados desde el año 1984 a lo que va del año 2020. Los años donde se registró mayor producción de documentos relacionados a estos conceptos es de 2018 al año 2020 con un total de 82 documentos, el artículo de 1984 aborda el tema de innovación en los servicios por medio de la tecnología que prevalecía en esa época y no se registran en los siguientes años documentos hasta 2005, 2006 y 2007 con un documento en cada año, a partir del año 2011 comienza a darse una mayor producción de trabajos.

Los países que registran mayor producción de acuerdo con los resultados son: Australia, Alemania, Reino Unido, Italia, Portugal, Canadá, para el caso de México solo se tiene un registro. En las siguientes figuras se presentan el tipo de documento y áreas del conocimiento de la producción generada con los conceptos “Transformación Digital” y "Servicios Públicos”.

Figura 4. Documentos relacionados con los conceptos “Transformación Digital” y "Servicios Públicos”.

Documents by type

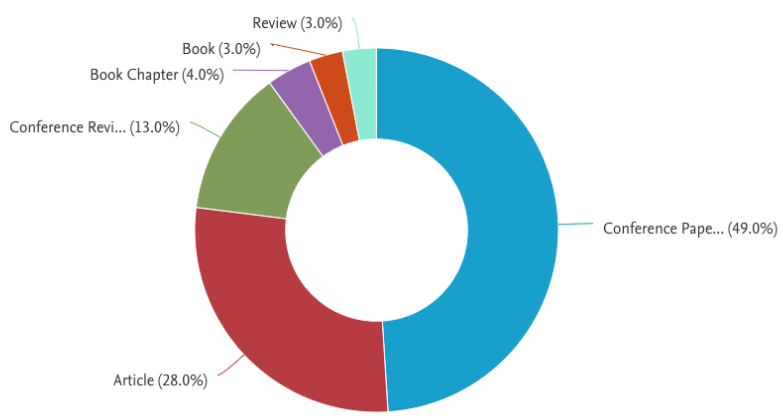

Fuente: Scopus (2020)

De acuerdo con la figura anterior el $49 \%$ de los documentos relacionados con ponencias en conferencias, mientras que el $28 \%$ son artículos y el $4 \%$ capítulo de libro; de estos resultados se tomaron como fuente para el estudio las, ponencias, artículos y capítulos de libro. 
Figura 5. Documentos relacionados con los conceptos “Transformación Digital” y "Servicios Públicos por áreas de conocimiento.

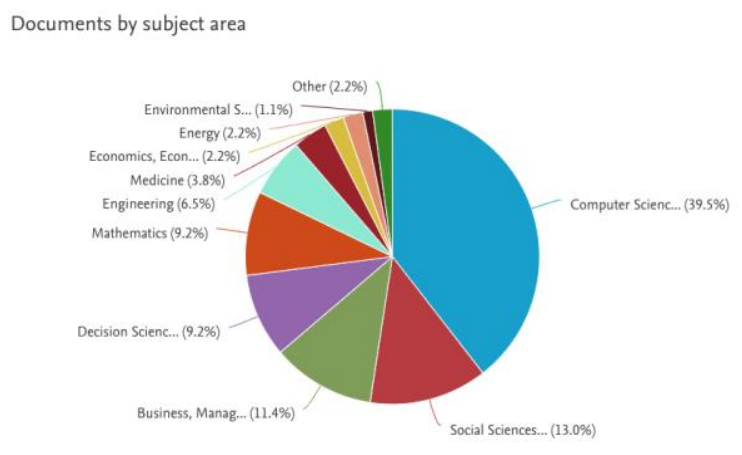

Fuente: Scopus (2020)

De la figura 5 se destaca que las ciencias de la computación representan el $39.5 \%$ de los documentos asociados a los conceptos principales de este estudio, el $24.4 \%$ se ubican en las ciencias sociales, negocios y administración. Para efectos del estudio de éstas se consideraron los que abordaban las dimensiones: estrategia, capacidades tecnológicas, gestión y cambio.

A partir del análisis anterior y aplicando los filtros señalados líneas arriba, se utilizaron 27 documentos de los cuáles 16 corresponden a los conceptos "Cuarta Revolución Industrial" y "Transformación Digital" y 11 documentos a "Transformación Digital” y "Servicios Públicos". Con esto se puede apreciar que los modelos de transformación digital que se revisaron en el apartado de revisión teórica y propuestos por Westerman, McAfee y Bonnet (2014) y (nae, 2018) tiene los elementos y las dimensiones que facilitan el desarrollo de los procesos de transformación en las organizaciones.

Así mismo de este análisis, se puede advertir que son temas relativamente incipientes pero que a partir de los últimos dos años han tenido un crecimiento exponencial, derivado del uso intensivo de la tecnología, sus aplicaciones digitales y factores emergentes como la contingencia sanitaria provocada por la propagación del virus SARS-CoV-2 (Covid-19) que en el año 2020 intensificó que las empresas y organizaciones incorporarán a sus procesos alta tecnología y la digitalización.

También el análisis permitió identificar que, en países latinoamericanos, como en el caso de México es baja la producción académica relacionado transformación digital con enfoque en los servicios públicos, pero ello representa una oportunidad para posicionar más estos temas como objeto de investigación y además como prácticas para que el gobierno e instituciones públicas incorporen más servicios de manera digital. 


\section{CASO DE ESTUDIO}

En las Instituciones de Educación Superior en México públicas o privadas, los procesos de transformación tecnológica están más enfocados a las actividades sustantivas como lo son la docencia, la investigación, la extensión y dejando para un segundo o tercer lugar las actividades de administración y de gestión, cuyos procesos se realizan de manera manual y los clientes tienden acudir a múltiples ventanillas para realización de trámites personales, dedicando tiempo y recursos en desplazamientos a efecto de realizar trámites que son necesarios como parte de la vida pública o bien en la mejora de sus condiciones laborales según sea el caso.

Para este caso la institución analizada es la Universidad de Guanajuato en particular la Dirección de Recursos Humanos que ha venido modernizando su tecnología y transformando procesos de manera digital. Dicha institución en su Plan de Desarrollo Institucional 2021-2030, en el eje Gestión Institucional, contempla como estrategia, la Universidad Digital, que establece que: "se continuará con la estrategia de transformación digital, que incluye la política de digitalización de trámites, archivos y expedientes institucionales; así como la automatización de los procesos, procedimientos y servicios, con el objetivo de hacer más eficiente la gestión" (PLADI 2021-2030, p.66).

A partir de lo anterior la oficina de Recursos Humanos de la Institución estudiada atiende a una comunidad de trabajadores y jubilados universitarios que asciende a más de 9000 personas, distribuidas en 13 municipios del estado de Guanajuato en donde la Universidad tiene presencia; los servicios más relevantes que ofrece al personal están relacionados con trámites de prestaciones laborales, solicitud de documentos como constancias laborales, emisión de documentos oficiales en materia laboral, créditos a trabajadores de base, entre otros.

Los trámites de los servicios se realizaban en las oficinas de enlaces de recursos humanos de las sedes foráneas y sede central, por tal motivo y alineados al Plan de Mejora, desde hace 5 años se inició con un plan de trabajo para modernizar la tecnología y migrar la atención de los servicios de manera digital. Para el desarrollo del plan de trabajo en todo momento se procuró que la plataforma ofreciera servicios de calidad, eficiencia, innovación, simplificación,

El plan de trabajo tuvo el apoyo y respaldo de la Alta Dirección, se realizó una inversión para adquisición de tecnología, se capacitó al personal vinculado a la realización del proyecto, se contrataron servicios de consultoría especializada con enfoque a experiencia en el usuario, esta fue la primera etapa del plan de trabajo. En la segunda etapa se analizaron y se identificaron los trámites que pudieran a migrar a formato digital, posteriormente se comenzó con la arquitectura y desarrollo, se realizaron pruebas piloto, se aplicaron ajustes.

Después de ello, se comenzaron con campañas de comunicación ante la comunidad de trabajadores informando de los trámites y el procedimiento que se realizan de manera digital, siendo 
estos: prestaciones laborales y constancias laborales -de éstas últimas se elaboran 7 tipos de documentos-. El portal digital donde se encuentran estos trámites se denominó ventanilla virtual, la cual se encuentra alojada en la intranet institucional.

Como última etapa del plan, fue la puesta en operación de la ventanilla, de acuerdo con nuestro informante -el responsable técnico del proyecto- destaca que al inicio no tuvo la aceptación esperada, por lo que se realizaron más campañas de difusión, videos tutoriales. Fue hasta que comenzó la pandemia por la propagación del COVID-19, a partir de las medidas tomadas por las autoridades sanitarias, que el uso de los servicios ofrecidos por la ventanilla virtual se incrementó.

\section{DISCUSIÓN}

La transformación digital como se ha señalado requiere de un fuerte liderazgo y alto compromiso de la organización para que ésta permee en toda la organización. En el caso descrito líneas arriba se puede apreciar que las dimensiones vertidas en el apartado teórico se encuentran presentes. En lo que respecta a la estrategia y liderazgo, en el caso, se señaló que existe un Programa de Mejora a la Gestión que contempla la Transformación Digital y desde luego esto ha sido posible por que la alta dirección lo ha puesto como prioridad institucional y se ha involucrado en ello, tienen una visión transformadora del futuro digital. En lo que respecta a las capacidades digitales, en la organización se puede apreciar, que ha invertido de manera sostenida en infraestructura tecnológica, sin esto no es posible desarrollar la digitalización de los servicios señalados.

Por lo anterior, se puede señalar a partir de la revisión sistemática de la literatura que la transformación digital a partir de la implantación de tecnología en los servicios públicos es indispensable contemplar la estrategia, las capacidades digitales, y la gestión del cambio, así mismo el modelo de transformación desarrollado por NAE(2018) se adapta en el proceso transformación digital de los servicios que brinda la ventanilla virtual de servicios de Recursos Humanos de la institución universitaria. Se antepone al cliente buscando atender las necesidades y brindar el servicio con calidad. Como se ilustró en el caso, el desarrollo de los servicios que ahora se ofrecen de manera digital los tiempos de respuesta se redujeron y se evita el traslado de los clientes, se simplificaron los trámites; la tecnología se puede quedar de manera permanente e incluso mejorar; es una innovación en el servicio; se reitera el compromiso del equipo de trabajo que destaca por su capacidad y conocimiento técnico para ejecutar la transformación digital.

Lo anterior generó un cambio en los procesos, al principio de acuerdo con nuestros informantes, generó confusión, en algunas personas incomodidad por que no usan con frecuencia la tecnología para realizar este tipo de trámites, sobre todo por que la mitad de la plantilla de personal de la institución 
son de la generación que ha aprendido a utilizar la tecnología y no nacido con ella. Pero el desarrollo de tutoriales sobre el uso de las aplicaciones facilitó la adaptación de los usuarios.

\section{CONCLUSIONES.}

Con este trabajo se pone de relieve que la transformación digital está presente en todas las organizaciones (públicas o privadas) y que se ha intensificado cada vez más en los años recientes, producto de la cuarta revolución industrial. Dicho cambio ha generado nuevos modelos de negocio, nuevas formas organizacionales y ha potenciado la innovación en particular la de los servicios. En este contexto, resulta necesario desarrollar competencias adecuadas para los nuevos empleos de hoy y mañana. Asimismo, es necesario que las organizaciones asuman su deber de integrarse al cambio digital.

El trabajo se focalizó en el estudio de transformación digital en los servicios públicos, en específico, el desarrollo de ventanilla única virtual, este enfoque brinda la posibilidad de potenciar la digitalización y trascender significativamente en los usuarios que integran una comunidad, en este caso la comunidad universitaria.

El análisis sistémico de la literatura permitió identificar las dimensiones requeridas en los procesos de transformación digital en los servicios públicos, además contribuyó para que en el estudio se estableciera la relación entre la teoría y los datos obtenidos a través del trabajo de campo realizado en la organización, es importante señalar que las metodologías cualitativas buscan explicar un problema y las posibles soluciones. Es importante destacar que será necesario comprobar el funcionamiento del modelo, para ello se requerirá realizar estudios con el uso de otras estrategias metodológicas que permitan contar correlaciones entre las dimensiones que se plantearon en el modelo propuesto que, como ya se ha referido se desarrolló a partir del análisis de la revisión de la literatura, ante tal circunstancia, se deberán diseñar instrumentos de evaluación a partir de los componentes que integran el modelo.

Por último, este estudio es una pequeña contribución para el estudio de transformación digital en los servicios públicos, y como se describió en el apartado de revisión de la literatura, en países considerados en vías de desarrollo, tal es el caso de México es incipiente. 


\section{REFERENCIAS}

CAVE, M., FLORES-ROUX, E. Posibles beneficios de la economía digital para México. 2017. http://ceeg.mx/publicaciones/Posibles-Beneficios-de-la-Economia-Digital_paraMexico_2017.01.27.pdf

CHRISTENSEN, C., RAYNOR M. E. y MCDONALD R. Waht is Disruptive Innovation? Harvard Bussines Review, 2015: 44-53

CUENCA-FONTBONA, J.; MATILLA, K.; COMPTE-PUJOL, M. Transformación digital de los departamentos de relaciones públicas y comunicación de una muestra de empresas españolas. Revista de Comunicación, v. 19, n. 1, p. 75-92, 10 mar. 2020.

https://doi.org/10.26441/RC19.1-2020-A5

CASTRO, L., TAMAYO M., J. A., ARANGO, M.D., BRANCH J.W., Burgos D. Digital Transformation in Higher Education Institutions: A Systematic Literature Review. Sensors, 20 (11), 2020: 123. https://www.mdpi.com/1424-8220/20/11/3291

DALKIR, K. Knowledge application. Knowledge management in theory and practice. Burlington, MA: Elsevier, 2017: 145-175.

DING, W., LIANG, P., TANG, A., y VLIET, H.V. Knowledge-based approaches in software documentation: A systematic literature review, Information and Software Technology, n. 56, 2014: 545567.

GOBIERNO DE MÉXICO: México Digital. Estrategia Digital Nacional, 2020. https://www.gob.mx/mexicodigital

GOBBLE, M.M. Digital strategy and digital transformation. Management, n. 61, 2018: 66-71.

HERNÁNDEZ, L. México avanza dos peldaños en Índice Mundial de Competitividad Digital. DPL News, 2019. https://digitalpolicylaw.com/mexico-avanza-dos-peldanos-en-indice-mundial-decompetitividad-digital/

GHOBAKHLOO, M. Industry 4.0, digitization, and opportunites for sustainability. Journal of Cleaner Production, 2020: 252

https://doi.org/10.1016/j.jclepro.2019.119869

HARZING, AW. Publish or Perish, 2017: https://harzing.com/resources/publish-or-perish

HOFMANN, S., S., ØYSTEIN, A.M. BRACCINI, S. Za. The public sector's roles in the sharing economy and the implications for public values. Government Information Quarterly, v.36, n.4, 2019: 101399. https://doi.org/10.1016/j.giq.2019.101399

JANSSEN, M., ESTEVEZ, E. Lean government, and platform-based governance-Doing more with less. Government Information Quarterly, vol. 30, n.1, 2013: 51-58. https://doi.org/10.1016/j.giq.2012.11.003 
KARLSSON, A., LARSSON, L., RÖNNBÄCK, A. Product-service system innovation capabilities: linkages between the fuzzy front end and subsequent development phases. International Journal of Production Research, vol. 56, n.6, 2018: 2218-2232, https://doi.org/10.1080/00207543.2017.1365181

LOMBARDERO, L. Trabajar en la era digital. Tecnología y competencias para la transformación digital. LID Editorial. 2015.

MERGEL, I. Open innovation in the public sector: drivers and barriers for the adoption of Challenge.gov. Public Management Review, v.5, n.20, 2018: 726-745.

MERGEL, I., EDELMANN, N., HAUG, N. Defining digital transformation: Results from expert $\begin{array}{llllll}\text { interviews. Government Information } & \text { Quarterly, } & \text { v. } & \text { 4, n.36, }\end{array}$ 101385. https://doi.org/10.1016/j.giq.2019.06.002

NAE Nae doing ahead: Claves para un nuevo modelo de transformación digital. 2018. https://nae.global/claves-para-un-nuevo-modelo-de-transformacion-digital/

OECD. Measuring the Digital Transformation: A Roadmap for the Future, OECD Publishing, Paris, 2019 https://doi.org/10.1787/9789264311992-en.

SCHWAB, Klaus. La cuarta revolución industrial. México: Debate, 2016.

SKALEN, P., KARLSSON, J., ENGEN, M. y MAGNUSSON, P. R. Understanding Public Service Innovation as Resource Integration and Creation of Value Propositions. Australian Journal of Public Administration, v. 77, n. 4, 2017: 700-714. doi:10.1111/1467-8500.12308

UNIVERSIDAD DE GUANAJUATO. Plan de Desarrollo Institucional de la Universidad de Guanajuato 2021-2030, https://www.ugto.mx/planeacion/images/pdf/universidad-de-guanajuatopladi-2021-2030.pdf

WANG, L., LI, Z. Knowledge flows from public science to industrial technologies. The Journal of Technology Transfer, v. 44, n.3. 2019. https://doi.org/10.1007/s10961-019-09738-9

WEILL, Peter, WOERNER, STEPHANIE L. Which Digital Business Model Is Best for Your Future? Book: What's Your Digital Business Model? Six Questions to Help You Build the Next-Generation Enterprise. Harvard Business Press, 2018: 37-64

WESTERMAN, G., MCAFEE A. and BONNET, D. Leading Digital- Turning technology into business transformation. Boston, MA. Harvard Business Review Press. 2014.

WORLD BANK World Development Indicators: Investment in telecoms with private participation. 2016. https://databank.worldbank.org/home.aspx

YE, H.J. y KANKANHALLI, A. Value Cocreation for Service Innovation: Examining the Relationships between Service Innovativeness, Customer Participation, and Mobile App Performance. Journal of the Association for Information Systems, v.21, n. 2, 2020. 292-311. doi: 10.17705/1jais.00602

YIN, R. K. Case Study Research. Design and Methods. Thousand, Oaks, CA: SAGE Publications. 1984. 